\title{
Performance Analysis of CDL-impaired Multi-Core Fiber Transmission
}

\author{
Akram Abouseif $^{1}$, Ghaya Rekaya-Ben Othman ${ }^{2}$, Oussama Damen ${ }^{3}$ \\ ${ }^{1}$ MIMOPT Technology, Paris, France \\ ${ }^{2}$ Telecom Paris, Institut Polytechnique de Paris, Paris, France \\ ${ }^{3}$ Electrical and Computer Engineering, University of Waterloo, Ontario, Canada
}

Email address:

akram.abouseif@mimopt.com (A. Abouseif), ghaya.rekaya@telecom-paris.fr (G. Rekaya-Ben O.), mdamen@uwaterloo.ca (O. Damen)

\section{To cite this article:}

Akram Abouseif, Ghaya Rekaya-Ben Othman, Oussama Damen. Performance Analysis of CDL-impaired Multi-Core Fiber Transmission. American Journal of Optics and Photonics. Vol. 9, No. 3, 2021, pp. 39-50. doi: 10.11648/j.ajop.20210903.12

Received: September 14, 2021; Accepted: October 12, 2021; Published: November 23, 2021

\begin{abstract}
Single-mode fibers have reached a critical point in terms of optical communication capacity. Space division multiplexing (SDM) is one of the most promising candidates for increasing optical fiber capacity. SDM allows the propagation of multiple spatial channels where the paths could be multiple cores in a multi-core fiber (MCF). The transmission performance of MCFs is impaired by a non-unitary effect known as Core Dependent Loss (CDL). Multiple-input multiple-output (MIMO) technology is an effective solution to improve the transmission performance of MCFs. However, it can increase the system cost. Several techniques, such as core scrambling and Space-Time (ST) coding, have been proposed to mitigate CDL. This paper focuses on the analysis of the MCF transmission performance of different schemes. Our analysis concerns the derivation of an upper bound of the error probability by applying Maximum Likelihood (ML) and Zero-Forcing (ZF) decoders at the receiver. We also evaluate the performance of both core scrambling and ST coding systems. We prove that the ZF decoder offers similar performance to the ML decoder and confirm this with simulation results. Finally, to consider the cost factor of applying MIMO techniques, low complexity solution is proposed by combining core scrambling and ST codes using the sub-optimal ZF decoder and show performance close to the Gaussian channel.
\end{abstract}

Keywords: Fiber Optics Communications, MIMO, Space Division Multiplexing, Space-Time Coding

\section{Introduction}

Optical fiber communication systems have undergone several evolutions over the last few decades. All the degrees of freedom that single-mode fiber (SMF) can offer have been deployed, such as wavelength, amplitude, phase and polarization. The currently deployed SMF cannot keep up with the exponential increase in Internet demand. Space Division Multiplexing (SDM) is the most promising solution to increase the transmission capacity of optical fiber. In this system, capacity is multiplied by the number of spatial paths where each path carries independent data streams. SDM is provided by multi-mode fibers (MMF), which use more than one linear polarization (LP) mode in a single core, or by multi-core fibers (MCF), where each core carries a single mode or a few modes in the same cladding [1]. In this work, we are interested in evaluating the performance of MCFs.
The addition of a new multiplexing capacity introduces a critical challenge, namely crosstalk between neighboring cores. The effects of crosstalk come from adjacent cores, where several studies have been carried out to evaluate the factors that influence the crosstalk level [2-4]. A model for the temporal variation of crosstalk has been proposed using coupled-mode theory (CMT) and a stationary Brownian motion for the phase shifts introduced at each central point between the phase-matching points [5]. However, in our study, we apply the coupled-power theory (CPT), which provides the mean value of the crosstalk over time, allowing fast and accurate estimation of the crosstalk, as done by M. Koshiba et al. [6]. In addition, core misalignment has been considered a second factor affecting the performance of the MCF transmission system. The loss due to misalignment is caused by in-line components such as connectors and fan- $\{$ In/Out $\}$ devices [7].

The different crosstalk and misalignment losses in each core 
induce a Core Dependent Loss (CDL) [8], which is a non-unitary effect limiting the transmission capacity. CDL has an effect similar to Polarization Dependent Loss (PDL) on the polarization multiplexing (PolMux) system and Mode Dependent Loss (MDL) on MMF, which causes performance degradation. A statistical study on the MDL model and its effects on MMF have been carried out in several works $[9,10]$. Here, we focus on CDL, which has different properties from MDL and is based on the channel model of MCF. In our previous work [8], inspired by the work done in [10], we have derived a theoretical MCF channel model that takes into account the CDL effect. In the present paper, we apply this theoretical model to analyze the transmission performance of the MCF.

MCF can be divided into a weak-coupled fiber and a strong-coupled fiber. The privilege of the weak-coupled fiber is the ability to avoid using full MIMO processing at the receiver side. However, even for the weak-coupled regime, full MIMO processing provides transmission performance enhancement. For example, in [11], the authors demonstrated that full MIMO processing improves the range by $8 \%$ in weak-coupled MCFs. Therefore, in this work, we consider MIMO processing for both weak-coupled and strong-coupled regimes.

The system performance can be enhanced by applying the core scrambling technique that reduces the CDL by averaging the losses over the different cores [8]. Yet, the CDL level is not completely removed. Therefore, inspired by the works done on the PDL and MDL mitigation over PolMux and MMF systems, respectively, we propose in this paper Space-Time (ST) coding to mitigate the CDL in MCF systems. We show that ST codes can completely mitigate the CDL. In addition, we study the performance of an uncoded system with and without core scrambling and ST codes. We derive an upper bound of the error probability, taking into account the Maximum Likelihood (ML) and Zero-Forcing (ZF) decoding schemes. We prove analytically and show by simulations that ML and ZF have a similar Gaussian behavior using core scrambling in the transmission system. Moreover, to make ST coding solutions applicable to a larger number of cores, low complexity decoding solution is required. We propose a combination of core scrambling and ST coding; this technique significantly reduces the decoding complexity using the sub-optimal ZF decoder and achieves performance close to the optimal ML decoder.

The remainder of this paper is organized as follow. In Section 2, we describe the SDM transmission system and specify the main channel impairments coming from the crosstalk and the misalignment loss. In Section 3 we derive the upper bound error probability of the uncoded system for ML and ZF detection schemes. We devote Section 4 to introduce core scrambling strategy to mitigate the CDL and illustrate the benefit brought by using the core scrambling. In Section 5, we show that ML and ZF have similar performance thanks to core scrambling. In Section 6, we analyze the coded MCF transmission system based on ST coding scheme. First, we show that ST completely mitigates the CDL considering ML decoder at the receiver side. Then, we relate $\mathrm{ZF}$ decoding performance to the Orthogonal Defect
Factor (ODF) of coded system. Section 7 is dedicated to proposing low complex solutions by combining core scrambling and ST coding over MCF system. Finally, Section 8 concludes the paper.

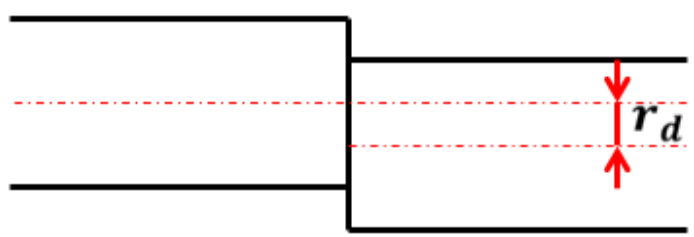

Figure 1. Transverse Misalignment.

\section{Multi-Core Fiber System Model}

The existence of cores in the same cladding allows the transfer of energy between the cores, causing linear crosstalk, which can be estimated based on the CPT. The average crosstalk between core $n$ and $m\left(X T_{n, m}\right)$ is estimated by Eq.(1) and Eq. (2) for homogeneous and heterogeneous cores, respectively [6]. $\beta$ and $\Delta \beta$ are the propagation constant and the difference between the two cores. $k_{n, m}$ is the coupling coefficient. $d$ is the correlation length between the bending and twisting effects on the fiber [6]. $L$ and $\Lambda$ are respectively the fiber length and the core pitch.

$$
\begin{aligned}
& X T_{n, m}=\frac{2 k_{n m}^{2} R_{b}}{\beta^{2} \Lambda} L \\
& X T_{n, m}=\frac{2 k_{n m}^{2}}{\Delta \beta^{2} \Lambda} L
\end{aligned}
$$

Further, the imperfection of the optical fiber at the splicing points, the connectors, and the Fan- $\{\mathrm{In} / \mathrm{Out}\}$ devices along the transmission link induces different misalignment losses in each core such as transverse, longitudinal, and angular misalignment $[12,13]$. In [14], the authors showed that the transverse misalignment loss $(\alpha)$, as shown in Figure 1, has the most significant impact on the propagating losses. Therefore, we assume that the transverse misalignment is the only source affecting the propagating signal, which is defined as [13]:

$$
\alpha=\exp \left(-b r_{d}^{2}\right)
$$

where $r_{d}$ is the fiber transverse displacement in the $x$ and $y$ directions, and $b$ depends on the fiber mode fields radius before and after the splicing [13].

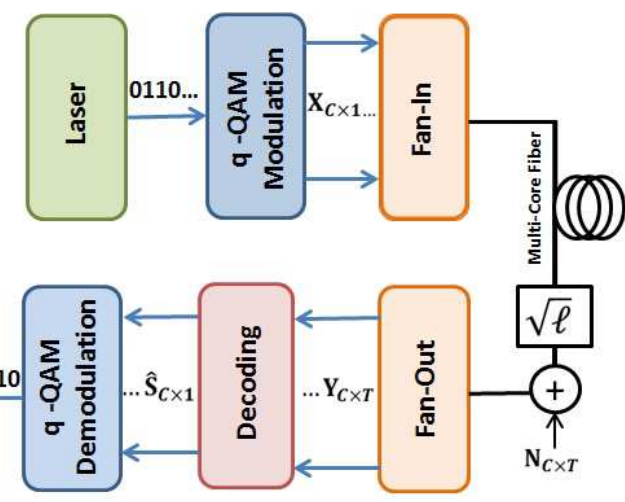

Figure 2. MCF transmission scheme. 
In this work, we consider an MCF transmission with a linear propagation regime. In fact, one of the privileges of MCF deployment is to avoid entering the non-linearity while increasing the capacity of the transmission system. Moreover, we assume a complete mitigation of the dispersion effect by applying a time domain equalizer (TDE) or a frequency domain equalizer (FDE) as well-known techniques for compensating the differential mode group delay (DMGD) [15-17]. Considering an MCF system with $C$ cores, each allows the propagation of the fundamental mode with a single polarization to focus on the CDL effect. As shown in Figure 2, the signal received from the linear MIMO channel over the duration of the $T$ symbols can be given by:

$$
\mathrm{Y}_{C \times T}=\mathrm{H}_{C \times C} \mathrm{X}_{C \times T}+\mathrm{N}_{C \times T}
$$

where $\mathrm{H}$ is represented as [10]:

$$
\mathrm{H}=\sqrt{\ell} \prod_{k=1}^{K}\left(\mathrm{~T}_{k}\left(\mathrm{H}_{X T}\right)_{k} \mathrm{M}_{k}\right)
$$

with $\mathrm{X}_{C \times T}$ and $\mathrm{Y}_{C \times T}$ are the emitted and the received vectors, respectively, where $T=C$ for the $\mathrm{ST}$ coded system and $T=1$ for the uncoded system. $\mathrm{H}$ is the equivalent $\mathrm{MCF}$ channel matrix, which is a concatenation of $K$ fiber sections. $\left(\mathrm{H}_{X T}\right)_{k}$ represent the square crosstalk channel matrices. As an example, the crosstalk channel matrix for 7-core MCF is represented as:

$$
\mathrm{H}_{\mathrm{XT}}=\left[\begin{array}{cccc}
X T_{1} & X T_{1,2} & \cdots & X T_{1,7} \\
X T_{2,1} & \ddots & & X T_{2,7} \\
\vdots & & \ddots & \vdots \\
X T_{7,1} & X T_{7,2} & \cdots & X T_{7}
\end{array}\right]
$$

where $X T_{i}=1-\sum_{n \neq m} X T_{n . m}$.

The different misalignment losses are defined by diagonal matrices $M_{k}$ with different loss values at each core $\left(\operatorname{diag}\left(\alpha_{1}, \ldots, \alpha_{C}\right)\right)[8]$. As presented in [13], we assume that the misalignment loss values are random Gaussian variables of zero mean and standard deviation equal to the percentage of the core radius $\left(\sigma_{x, y}=\% r_{c}\right.$, where $r_{c}$ is the core radius), in order to validate Eq.(3). $T_{k}$ is a diagonal matrix with uniformly distributed random phase inputs $\exp \left(j \varphi_{c}\right)$ where $\varphi_{c}$ $\in[0,2 \pi]$ that represent modal noise due to random changes of the phase constants induced by strain, temperature variation $[10,18] . \ell=\frac{C}{\sum_{i=1}^{C} \lambda_{i}}$ is a normalization factor used to compensate for link loss such that $\operatorname{Tr}\left(\mathrm{HH}^{H}\right)=C$ (with $\operatorname{Tr}($.) representing the trace of a matrix operator and $H^{H}$ is the conjugate transpose of $H), \lambda_{i}$ are the eigenvalues of the channel matrix $H$ [10]. Finally, $N$ is an additive white Gaussian noise matrix with i.i.d. inputs of mean zero and variance $N_{0}$. In a previous work [8], we modeled the MCF channel using the singular value decomposition as:

$$
H=U \Sigma V^{H}
$$

where $U$ and $V^{H}$ are random unit matrices and $\Sigma$ is the diagonal matrix of singular values where each element has a lognormal distribution which is a function of the core parameters and the level of misalignment. Furthermore, the $\mathrm{CDL}$ is defined as the ratio between the maximum eigenvalue $\left(\max \left\{\lambda_{i}\right\}\right)$ and the minimum eigenvalue $\left(\min \left\{\lambda_{i}\right\}\right)$ of $H H^{H}$ in $\mathrm{dB}\left(\mathrm{CDL}=10 \cdot \log _{10}(\varepsilon)\right.$, where $\left.\varepsilon=\max \left\{\lambda_{i}\right\} / \min \left\{\lambda_{i}\right\}\right)$.

\section{MCF Transmission Performance Analysis}

In this section, we analyze the ML and $\mathrm{ZF}$ detection performance over uncoded MCF transmission systems impaired by the CDL.

\subsection{Maximum Likelihood Detection}

The ML decoder provides the optimal data at the receiver side. The emitted vector X estimation should satisfy the ML criteria based on minimizing the Euclidean distance as follow:

$$
\widehat{X}_{M L}=\operatorname{argmin}_{X \in \mathcal{C}}\|\mathrm{Y}-\mathrm{HX}\|^{2}
$$

where $\mathcal{C}$ is the set of all possible transmitted vector of symbols. Furthermore, the pairwise error probability (PEP) to detect $\mathrm{X}_{2}$ given that $\mathrm{X}_{1}$ has been transmitted is written as [19]:

$$
\begin{gathered}
\operatorname{Pr}\left\{\mathrm{X}_{1} \rightarrow \mathrm{X}_{2} \mid \mathrm{H}\right\}=\operatorname{Pr}\left\{\widehat{\mathrm{X}}=\mathrm{X}_{2} \mid \mathrm{X}_{1}, \mathrm{H}\right\} \\
\operatorname{Pr}\left\{\mathrm{X}_{1} \rightarrow \mathrm{X}_{2} \mid \mathrm{H}\right\}=Q\left(\frac{\left\|\mathrm{HX}_{\Delta}\right\|}{\sqrt{2 N_{0}}}\right)
\end{gathered}
$$

where $X_{\Delta}=X_{2}-X_{1}$. To obtain an upper bound of the error probability, the PEP is averaged over all the channel realizations. The previous equation can be presented as:

$$
\operatorname{Pr}\left\{\mathrm{X}_{1} \rightarrow \mathrm{X}_{2} \mid \mathrm{H}\right\}=Q\left(\sqrt{\frac{\operatorname{Tr}\left(\mathrm{HX}_{\Delta} \mathrm{X}_{\Delta}^{H} \mathrm{H}^{H}\right)}{2 N_{0}}}\right)
$$

Replacing the matrix $\mathrm{H}$ by the corresponding channel model in (7), then, applying the permutation property of the trace. With the fact that $U$ is a unitary matrix, Eq. (11) is rewritten as:

$$
\operatorname{Pr}\left\{\mathrm{X}_{1} \rightarrow \mathrm{X}_{2} \mid \mathrm{H}\right\}=Q\left(\sqrt{\frac{\ell \operatorname{Tr}\left(\Omega \mathrm{V}^{\mathrm{H}} \mathrm{X}_{\Delta} \mathrm{X}_{\Delta}^{H} \mathrm{~V}\right)}{2 N_{0}}}\right)
$$

$\Omega$ is a diagonal matrix with eigenvalues elements $\lambda_{i}$. At high SNR, the closest neighbors where the distance between them is equal to $d_{\min }$ (the minimum distance of the corresponding $Q A M$ constellation) dominate the error probability. An upper bound can be expressed as [19]:

$$
P_{e} \leq N_{\min } Q\left(\sqrt{\frac{\ell \operatorname{Tr}\left(\Omega \mathrm{V}^{\mathrm{H}} X_{\Delta} X_{\Delta}^{H} \mathrm{~V}\right)}{2 N_{0}}}\right)
$$

where $N_{\min }$ is the kissing number (number of closest neighbors). Moreover, for all the words differences in the closest neighbors set has $\mathrm{X}_{\Delta} \mathrm{X}_{\Delta}{ }^{H}=d_{\text {min }}^{2}$ as proven in [20]. Lastly, by developing the trace(.) we obtain:

$$
P_{e} \leq N_{\min } Q\left(\sqrt{\frac{\ell d_{\min }^{2} \gamma_{0}}{2} \sum_{i} \sum_{j} \lambda_{i}\left|v_{i, j}\right|^{2}}\right)
$$

where $\gamma_{0}$ is the SNR, an upper bound of the above expression 
can be obtained by substituting each $\ell \lambda_{i}$ by the minimum over the maximum. In this case, the $\mathrm{V}$ factor is removed by applying the unitary property of $\mathrm{V}$.

$$
P_{e} \leq N_{\min } Q\left(\sqrt{\frac{d_{\min \gamma_{0}}^{2}}{2} \frac{\min \left\{\lambda_{i}\right\}}{\max \left\{\lambda_{i}\right\}}}\right)
$$

One substitutes $\max \left\{\lambda_{i}\right\}$ by the practical $\lambda_{\max }$, the one that dominates the upper bound on the error probability, which is obtained by applying the maximum level of the confidence interval. Thus, the upper bound can be rewritten as:

$$
P_{e} \lesssim N_{\min } E_{X}\left[Q\left(\sqrt{\frac{d_{\min }^{2} \gamma_{0}}{\lambda_{\max }} X}\right)\right]
$$

where $X=\min \left\{\lambda_{i}\right\}$. In [8], we proved that the eigenvalues $\lambda_{i}$ have lognormal distribution $\left(\lambda_{i}=\zeta_{i} \exp (Z)\right.$ where $\zeta_{i}$ is the crosstalk level and $Z \sim \mathcal{N}(\mu, \sigma)$ ). Moreover, the eigenvalue in each core has very low standard deviation $(\sigma \ll 1)$, thus the lognormal distribution can be assumed as normal distribution based on [21]. Lastly, since $\lambda_{i}$ are independent, the CDF of $X$ is given as $1-\left(1-F_{\lambda_{i}}(x)\right)^{C}$ where $F_{\lambda_{i}}(x)$ is the CDF of random variable $X$ (see Appendix. A). So, the PDF of $X$ is obtained as:

$$
f_{X}(x)=\frac{C}{\sigma \sqrt{2 \pi}}\left[\operatorname{erfc}\left(\frac{x-\mu}{\sigma \sqrt{2}}\right)\right]^{C-1} e^{-\left(\frac{x-\mu}{\sqrt{2} \sigma}\right)^{2}}
$$

As shown in Appendix. B, we derive Eq. (15) to obtain an upper bound error probability as:

$$
P_{e} \lesssim \widetilde{K} e^{-\frac{\lambda_{\min }^{2}}{2 \pi \sigma^{2}}} e^{-\pi \sigma^{2} \frac{d_{\min }^{2} \gamma_{0}}{\lambda_{\max }}}
$$

where $\widetilde{K}=K \frac{\pi \sigma}{\sqrt{2 C}}$.

\subsection{Zero-Forcing Detection}

The sub-optimal ZF decoder is the simplest linear decoder. The received signal $\mathrm{Y}$ is multiplied by the channel pseudo-inverse $G_{Z F}=\mathrm{H}^{H}\left(\mathrm{H}^{H} \mathrm{H}\right)^{-1}$ to remove the crosstalk in all the channels. The post-detection SNR of the ZF detection is expressed as $[22,23]$ :

$$
\gamma=\frac{\gamma_{0}}{\left[\mathrm{H}^{H} \mathrm{H}\right]_{i i}^{-1}}
$$

where $\gamma_{0}$ is the normalized received SNR. Given the optical channel matrix $\mathrm{H}$ in (7):

$$
\left[\mathrm{H}^{H} \mathrm{H}\right]^{-1}=\ell^{-1} \mathrm{~V} \Omega^{-1} \mathrm{~V}^{H}
$$

by substituting in Eq. (19):

$$
\gamma=\frac{\gamma_{0} \ell}{\sum_{i=1}^{C} \lambda_{i}^{-1}\left|u_{i l}\right|^{2}}
$$

Thus, the bit error probability with the $\mathrm{ZF}$ decoder is given by $a Q(\sqrt{\beta \gamma}$ where $a$ and $\beta$ depend on the constellation $(q-Q A M)[22]$ that can be expressed as:

$$
P_{e} \leq E[a Q(\sqrt{\beta \gamma})]
$$

$$
P_{e} \leq E\left[a Q\left(\sqrt{\frac{\ell \beta \gamma_{0}}{\sum_{i} \lambda_{i}^{-1}\left|u_{i l}\right|^{2}}}\right)\right]
$$

The expression is simplified to obtain an upper bound of the error probability as:

$$
\gamma=\frac{\ell \beta \gamma_{0}}{\sum_{i=1}^{C} \lambda_{i}^{-1}\left|u_{i l}\right|^{2}}>\frac{\beta \gamma_{0} \min \left\{\lambda_{i}\right\}}{\max \left\{\lambda_{i}\right\}}
$$

with the same methodology as the previous section by substituting $\max \left\{\lambda_{i}\right\}$ by the practical $\lambda_{\max }$ and $X=\min \left\{\lambda_{i}\right\}$, the upper bound error probability of the ZF detection is obtained by:

$$
P_{e} \lesssim a E_{X}\left[Q\left(\sqrt{\frac{\beta \gamma_{0}}{\lambda_{\max }} X}\right)\right]
$$

We notice that we end up with almost the same expression as the ML decoder. Thus, we develop the expectation with the same method as the previous section, which leads to:

$$
P_{e} \lesssim M e^{-\frac{\mu^{2}}{2 \pi \sigma^{2}}}\left[\pi \sqrt{\frac{\sigma^{2}}{2 C^{2}}} e^{-\frac{b}{4 a}} \operatorname{erfc}\left(\frac{1}{2} \sqrt{\frac{b}{a}}\right)\right]
$$

with $M=\frac{C a}{\sigma \sqrt{2 \pi}}, b=\frac{C \gamma_{0}}{\lambda_{\max }}$ and $a=\frac{C}{2 \pi \sigma^{2}}$.

\subsection{Simulation Results}

In order to validate the expressions obtained in (18) and (26), we present the transmission system performance of both $\mathrm{ML}$ and $\mathrm{ZF}$ detection through numerical simulations and compare them to the theoretical upper bounds. In Figure 3, we plot the bit error rate (BER) versus the signal to noise ratio $\mathrm{SNR}=E_{b} / N_{0}$, the simulation set up is based on the 7-core heterogeneous MCF structure (7-core Hetero-MCF) with three different type of cores (Black, Blue and Red) as shown in Figure 4. The core radius and refractive indices are $r_{c}$ and $n_{0}$, respectively. $\Delta$ is the relative refractive index differences between the core and 1st cladding. A $16-Q A M$ modulation is used at the transmitter side, and $100 \mathrm{~km}$ fiber link with no amplification consists of 300 fiber sections $(K=300)$ with $7 \% r_{c}$ misalignment level. The considered link parameters result in a moderate CDL level equal to $4 \mathrm{~dB}$. In Figure 3(a), we show the performance of the ML decoder. We notice that the error probability upper bound obtained in Eq.(18) is tight at low SNR and almost exact at high SNR, with an SNR penalty equal to $2.5 \mathrm{~dB}$ at $\mathrm{BER}=10^{-4}$ compared to the Gaussian channel $(\mathrm{CDL}=$ zero $)$. Also, in Figure 3(b), we plot the performance of the ZF decoder, the error probability upper bound is in agreement with the $\mathrm{ZF}$ performance with more SNR penalty equal to $4 \mathrm{~dB}$ at $\mathrm{BER}=$ $10^{-4}$ compared to the Gaussian channel and $1.5 \mathrm{~dB}$ different compared to the ML performance.

In this section, we derived an analytical upper bound error probability to predict the MCF transmission performance based on system setup. In the following sections, we show that ML and ZF decoders can have similar performances by applying the core scrambling in the transmission link. 


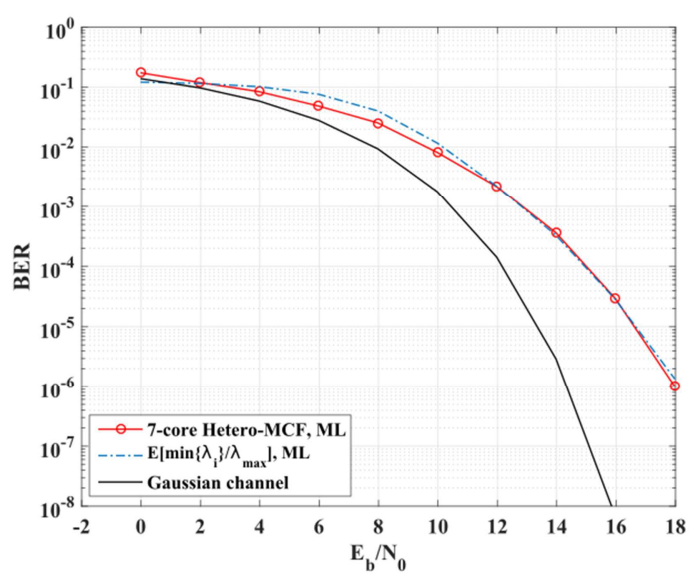

A. ML decoder

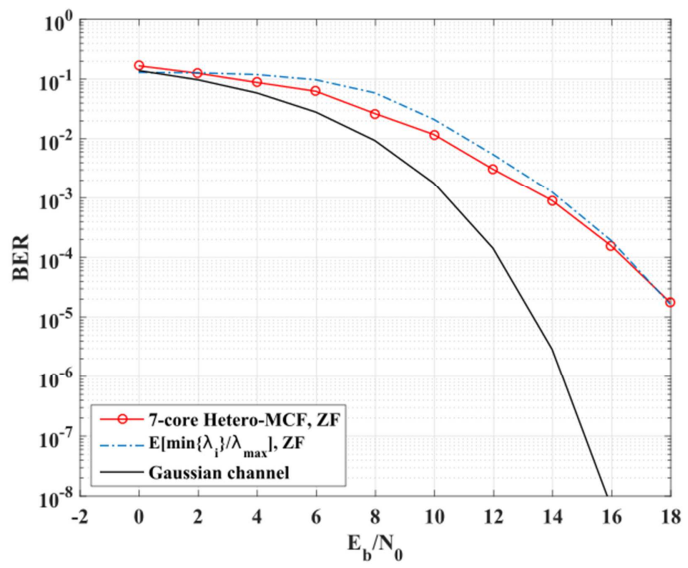

B. ZF decoder

Figure 3. Simulation and theoretical upper bound error probability for 7-core $M C F$ : A. ML decoder and B. ZF decoder, with $7 \% r_{c}$ misalignment level.

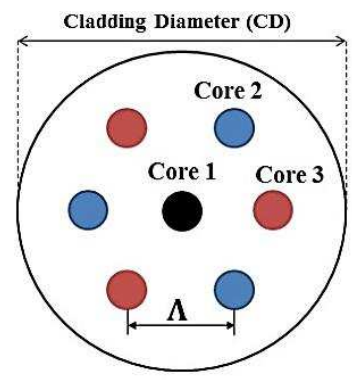

Core Structure Parameter

\begin{tabular}{|c|c|c|c|}
\hline Core & Core 1 & Core 2 & Core 3 \\
\hline$r_{c}\left[\mu_{m}\right]$ & 4.68 & 4.76 & 4.68 \\
\hline$n_{0}$ & 1.45 & 1.45 & 1.45 \\
\hline$\Delta[\%]$ & 0.305 & 0.338 & 0.388 \\
\hline
\end{tabular}

Figure 4. 7-core Hetero-MCF and the core structure parameter.

\section{MCF System Model with Core Scrambling}

It is important to keep the CDL value as low as possible in the transmission link to obtain a performance close to the AWGN channel. In the MCF link, each core is affected by different losses caused by different crosstalk and the level of misalignment. In Figure 5, we plot the average misalignment loss $\left(\alpha_{\text {avg }}\right)$ of each core type (core 1, core 2 and core 3 ), as mentioned in Figure 4, for several levels of offset $\left(\{0-10\} \% r_{c}\right)$ and fiber sections $K=300$. We notice that each core is affected by different losses with the same level of offset. In [8], it is reported that scrambling improves MCF system performance by reducing the CDL level. The function of core scrambling is illustrated in Figure 6. Assuming that MCFs have three different cores (black, red, and blue), we send three messages in each core $\left(S_{1}, S_{2}\right.$, and $\left.S_{3}\right)$. Then the scrambler is installed after $K_{s c r}$ section, and the signals are randomly or deterministically permuted and transmitted through the different cores. In this case, the signals are affected by the average losses induced by all cores.

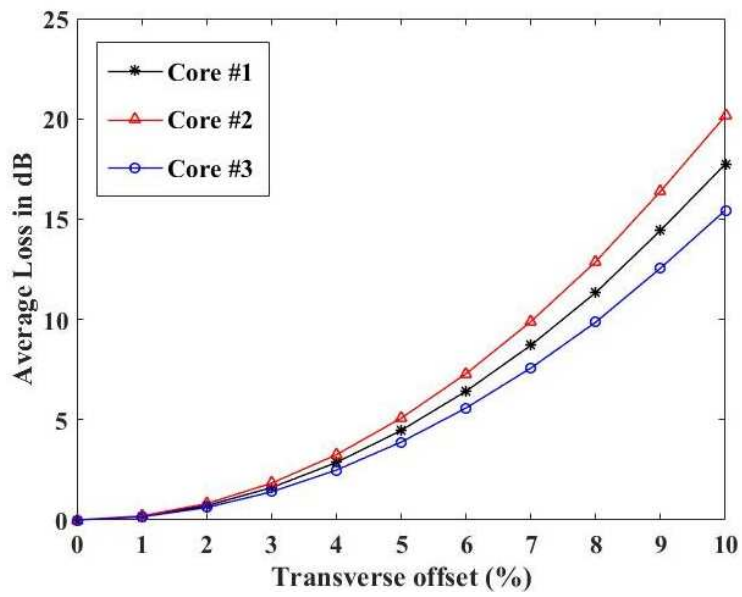

Figure 5. The average losses versus the transverse offset (\%) for three types of cores with fiber section number $=300$.

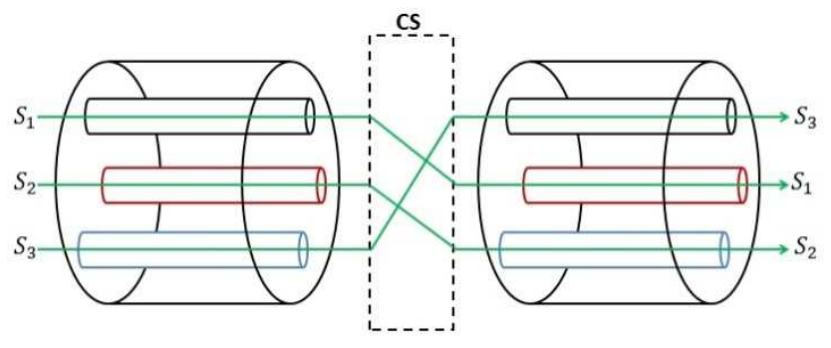

Figure 6. Core scrambling concept.

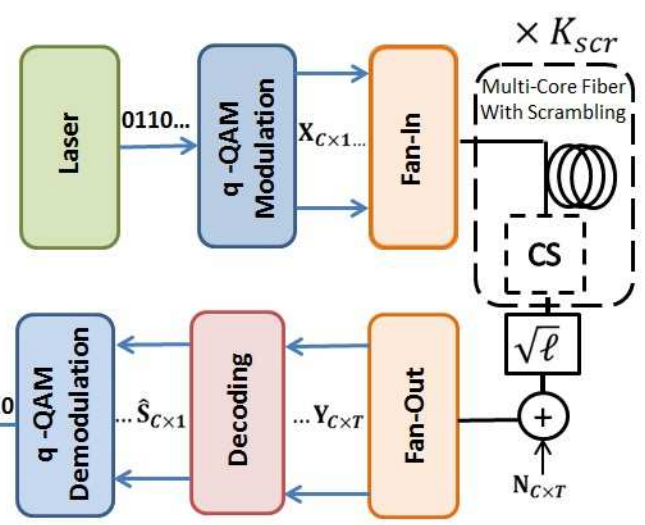

Figure 7. MCF transmission system with core scrambling.

The MCF transmission systems with installing the core scrambling as shown in Figure 7 can be expressed as:

$$
\mathrm{Y}_{C \times T}=\mathrm{H}_{C \times C} \mathrm{X}_{C \times T}+\mathrm{N}_{C \times T}
$$

where the MCF channel matrix $\mathrm{H}$ is expressed as: 


$$
\mathrm{H}=\sqrt{\ell} \prod_{k=1}^{K}\left(\mathrm{~T}_{k}\left(\mathrm{H}_{X T}\right)_{k} \mathrm{M}_{k} \mathrm{P}_{k}\right)
$$

$\mathrm{P}_{k}$ are the permutation matrices which represent the scramblers when $k$ is multiple of the scrambling period $K_{s c r}$ and identity matrices in other cases.

In Figures 8(a) and (b), we simulate $10^{5}$ channel realizations of the 7-core Hetero-MCF configuration with transverse offset equal to $7 \% r_{c}$ and $K=300$. We set $K_{s c r}$ to be equal to 50 , which corresponds to 6 core scramblers representing the optimal $\mathrm{CDL}$ reduction provided by

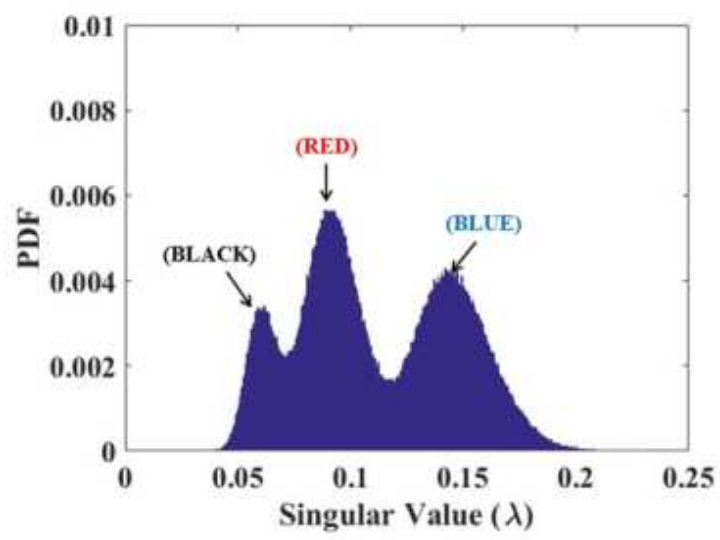

(a) Without scrambling deterministic scramblers, as proved in [8]. We plot the PDF of the singular values of the channel matrix for both cases with and without scrambling. In Figure 8(a), we notice three distributions that illustrate that the signals are affected by three different losses corresponding to the types of cores existing in the MCF as mention previously. In Figure 8(b), we can observe the effect of the scrambling since all the cores follow single distribution after averaging the losses, which causes less CDL level. Besides, this observation helps to derive a simple upper bound error probability in the following section.

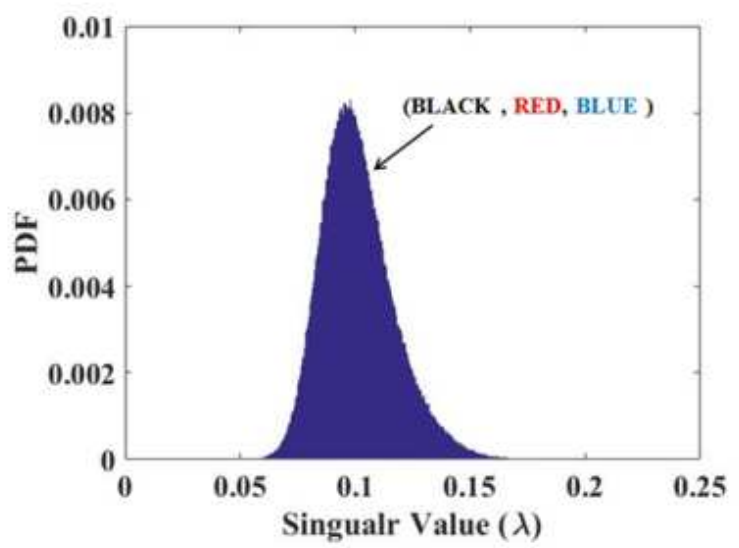

(b) With scrambling

Figure 8. The PDF of the singular values without and with core scrambling. Transverse offset $=7 \% r_{c}$ and fiber section number $=300, K_{\text {scr }}=50$.

\section{Performance Analysis of MCF System with Core Scrambling}

Through deriving an upper bound on error probability, in this section we proves analytically that the ML and the ZF decoders have similar performance after applying core scrambling in the transmission link.

\subsection{Maximum Likelihood Detection}

We begin our analysis by deriving an upper bound error probability of ML decoder with core scrambling. With the same methodology as Section 3, we start by Eq.(15) where both $\min \left\{\lambda_{i}\right\}$ and $\max \left\{\lambda_{i}\right\}$ are defined:

$$
P_{e} \leq N_{\min } Q\left(\sqrt{\frac{d_{\min }^{2} \gamma_{0}}{2} \frac{\min \left\{\lambda_{i}\right\}}{\max \left\{\lambda_{i}\right\}}}\right)
$$

Here, we take the privilege of the scrambling where all the cores have the same distribution as shown in Figure 8(b) and develop the last expression in a simpler method. The PDF of the $\min \left\{\lambda_{i}\right\}$ is defined in Eq. (17) $\left(X=\min \left\{\lambda_{i}\right\}\right)$. The CDF of $\max \left\{\lambda_{i}\right\}$ is defined as $F_{\lambda_{i}}(x)^{C}$. So, the PDF of $Y=\max \left\{\lambda_{i}\right\}$ is given as (see Appendix. A):

$$
f_{Y}(y)=\frac{C}{2^{C-2} \sigma \sqrt{2 \pi}}\left[1+\operatorname{erfc}\left(\frac{y-\mu}{\sigma \sqrt{2}}\right)\right]^{C-1} e^{-\left(\frac{y-\mu}{\sqrt{2} \sigma}\right)^{2}}
$$

given that $\sigma$ has a minimal value allows assuming that the PDF of $Y$ is also normally distributed.
Further, the mean values of $X$ and $Y$ are given by [24]:

$$
\begin{aligned}
& E[X]=\mu+\sigma E_{C} \\
& E[Y]=\mu-\sigma E_{C}
\end{aligned}
$$

where $E_{C}$ is defined as:

$$
E_{c}=\int_{-\infty}^{\infty} z \frac{d}{d z} F_{\lambda}(z) d z
$$

$F_{\lambda_{i}}(z)$ is the CDF of the eigenvalues. In [24], it has been shown that $E_{C}$ can be approximated by $E_{C} \sim \sqrt{2 \log (C)}$ which depends on the number of cores $C$ in the MCF.

From the previous results, we can define $\varepsilon=\max \left\{\lambda_{i}\right\}$ / $\min \left\{\lambda_{i}\right\}$ as lognormally distributed with $\mu_{\varepsilon}=E[Y]-E[X]$. Thus, the upper bound can be obtained when substituting the CDL by its mean $\frac{1}{\mu_{\varepsilon}}$ :

$$
\begin{gathered}
P_{e} \lesssim N_{\min } Q\left(\sqrt{\frac{d_{\min }^{2} \gamma_{0}}{2 \varepsilon}}\right) \\
\lesssim N_{\min } Q\left(\sqrt{\frac{d_{\min }^{2} \gamma_{0}}{2 \mu_{\varepsilon}}}\right)
\end{gathered}
$$

From the last expression, we observe that the MCF channel with scrambling is similar to the Gaussian performance with an SNR reduction equal to $\mu_{\varepsilon}$. This optical MIMO channel is entirely different from the wireless MIMO channel, which follows the Rayleigh model. 


\subsection{Zero-Forcing Detection}

In the case of the ZF decoder, the bit error probability is given as:

$$
P_{e} \lesssim E\left[a Q\left(\sqrt{\frac{\ell \beta \gamma_{0}}{\sum_{i} \lambda_{i}^{-1}\left|u_{i l}\right|^{2}}}\right)\right]
$$

which can be simplified by using the inequality:

$$
\frac{\ell \beta \gamma_{0}}{\sum_{i=1}^{C} \lambda_{i}^{-1}\left|u_{i l}\right|^{2}}>\frac{\beta \gamma_{0} \min \left\{\lambda_{i}\right\}}{\max \left\{\lambda_{i}\right\}}
$$

Then, we can directly substitute the ratio between $\min \left\{\lambda_{i}\right\}$ and $\max \left\{\lambda_{i}\right\}$ by the CDL $(\varepsilon)$ to obtain an upper bound error probability as follow:

$$
\begin{aligned}
P_{e} & \lesssim a E_{\varepsilon}\left[Q\left(\sqrt{\frac{\beta \gamma_{0}}{\varepsilon}}\right)\right] \\
& \lesssim a Q\left(\sqrt{\frac{\beta \gamma_{0}}{2 \mu_{\varepsilon}}}\right)
\end{aligned}
$$

We notice that the upper bound on the error probability of ML and ZF have the same expression (Eq.(34) and Eq.(37)) thanks to the core scrambling.

\subsection{Simulation Results of the Scrambling}

First, to illustrate the performance enhancement of the scrambling, in Figure 9 we plot the BER versus the SNR. We consider $16-Q A M$ modulation with ML or ZF decoder at the receiver side with the same fiber link parameter mentioned previously for core scrambling. We observe that using core scramblers in the transmission link decreases the SNR penalty to $0.5 \mathrm{~dB}$ instead of $2.5 \mathrm{~dB}$ for $\mathrm{ZF}$ decoder and instead of $4 \mathrm{~dB}$ for ML decoder without scrambling at BER $10^{-4}$. Second, in Figure 9 we validate the ML and ZF upper bound expressions in (34) and (37). We notice that ML and ZF detection have similar performance with $\mathrm{CDL}=1.8 \mathrm{~dB}$. This observation confirms our prediction through the derived expressions. Moreover, the upper bound on the error probability of ML and ZF detection show close estimation of the MCF system performance with core scrambling, since the different between the simulation and the upper bound is equal to $0.3 \mathrm{~dB}$ at $\mathrm{BER}=10^{-4}$.

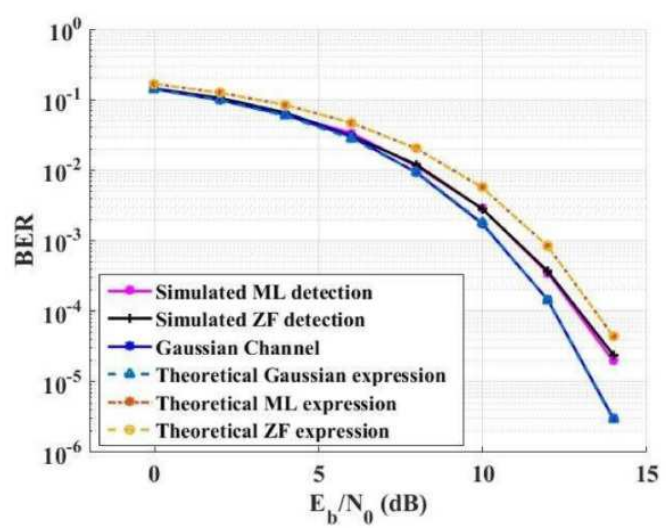

Figure 9. Simulation and theoretical upper bound of $M L$ and $Z F$ detection with core scrambling for 7-core Hetero- $M C F, C D L=1.8 d B$.
In conclusion, core scrambling is very efficient in reducing the CDL in an MCF transmission system. Averaging the losses allows all the spatial channels to follow a single distribution. The core scrambling gives the advantage to apply a sub-optimal ZF decoder offering very low complexity and similar performance as the ML decoder. The next sections will address the ability to reduce the remaining gap loss caused by CDL and enhance system performance.

\section{Space-Time Coding for CDL Mitigation}

In this section, we investigate the performance of Space-Time (ST) coding on MCF transmission systems, as shown in Figure 10. An analytical expression and simulation results are obtained to understand the performance enhancement of ST codes over MCF transmission systems.

\subsection{ST System Model}

In the study by E.-M. Amhoud [25], ST codes were applied experimentally to mitigate both the PDL in the two polarizations on PolMux systems and the MDL in several modes on MDM systems. ST coding technique is originally designed for wireless MIMO communications [26]. Here, we send multiple copies of independent $q-Q A M$ symbols in several cores at different time slots. At the receiver side, we can detect these copies and enhance data estimation since it will experience independent MCF channels.

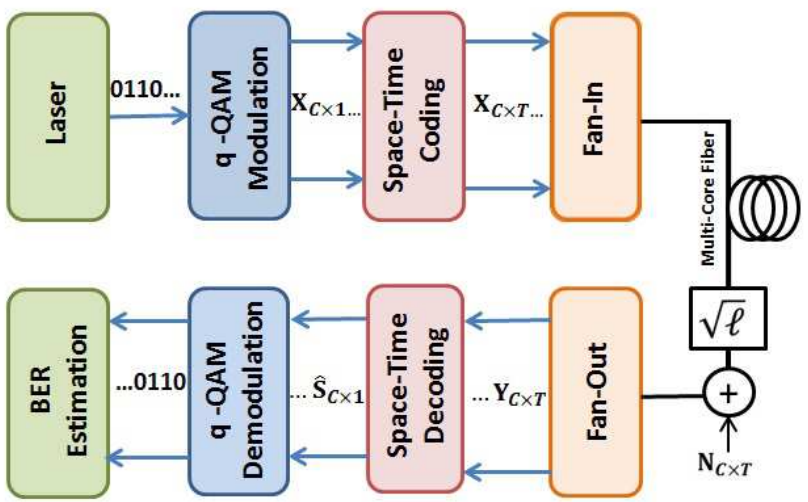

Figure 10. Space-Time coded MCF transmission system.

We consider Space-Time Block Codes (STBC) category where linear combinations of $q-Q A M$ symbols are created before sending them on $C$ cores and $T$ time slots. We apply a threaded algebraic space-time (TAST) code, which is known for the following properties: (i) placing each data symbol on a different core at each time slot while maintaining a minimum decoding delay, (ii) it has the same spectral efficiency as a spatially multiplexed system, and (iii) uniform average energy is transmitted per core [27]. We introduce the $7 \times 7$ TAST codeword matrix for 7-core Hetero-MCF as shown in Eq.(38). Each $s_{i}$ is a vector of 7 symbols where $i \in$ [1:7] and $\varphi=$ $\exp (j \pi / 12) \cdot f_{\ell}\left(s_{i}\right)=\sum_{k=1}^{7} x_{k}\left(\psi^{\ell-1} \theta\right)^{k-1}$, where $\ell \in$ $[1, \ldots, 7]$ with $\psi=\exp (j 2 \pi / 7)$ and $\theta=\exp (j \pi / 18) . \varphi$ and 
$\theta$ are calculated to ensure the full rate of the codeword [26]. In each codeword, we send $M$ symbols on $T=C$ time slots where $M=C T$; in the case of $7 \times 7$ TAST code $M=49$ symbols, which means that the maximum rate is achieved and equal to 7 symbols/channel use. Moreover, the coding matrix does not increase the energy of the transmitted symbols after the encoding, which satisfies the linear regime of fiber optics [10]. At the receiver side, the original symbols are estimated using ML detection to obtain the optimal performance.

$$
X_{T}=\frac{1}{\sqrt{7}}\left[\begin{array}{ccccccc}
f_{1}\left(s_{1}\right) & \varphi^{\frac{6}{7}} f_{2}\left(s_{7}\right) & \varphi^{\frac{5}{7}} f_{3}\left(s_{6}\right) & \varphi^{\frac{4}{7}} f_{4}\left(s_{5}\right) & \varphi^{\frac{3}{7}} f_{5}\left(s_{4}\right) & \varphi^{\frac{2}{7}} f_{6}\left(s_{3}\right) & \varphi^{\frac{1}{7}} f_{7}\left(s_{2}\right) \\
\varphi^{\frac{1}{7}} f_{1}\left(s_{2}\right) & f_{2}\left(s_{1}\right) & \varphi^{\frac{6}{7}} f_{3}\left(s_{7}\right) & \varphi^{\frac{5}{7}} f_{4}\left(s_{6}\right) & \varphi^{\frac{4}{7}} f_{5}\left(s_{5}\right) & \varphi^{\frac{3}{7}} f_{6}\left(s_{4}\right) & \varphi^{\frac{2}{7}} f_{7}\left(s_{3}\right) \\
\varphi^{\frac{2}{7}} f_{1}\left(s_{3}\right) & \varphi^{\frac{1}{7}} f_{2}\left(s_{2}\right) & f_{3}\left(s_{1}\right) & \varphi^{\frac{6}{7}} f_{4}\left(s_{7}\right) & \varphi^{\frac{5}{7}} f_{5}\left(s_{6}\right) & \varphi^{\frac{4}{7}} f_{6}\left(s_{5}\right) & \varphi^{\frac{3}{7}} f_{7}\left(s_{4}\right) \\
\varphi^{\frac{3}{7}} f_{1}\left(s_{4}\right) & \varphi^{\frac{2}{7}} f_{2}\left(s_{3}\right) & \varphi^{\frac{1}{7}} f_{3}\left(s_{2}\right) & f_{4}\left(s_{1}\right) & \varphi^{\frac{6}{7}} f_{5}\left(s_{7}\right) & \varphi^{\frac{5}{7}} f_{6}\left(s_{6}\right) & \varphi^{\frac{4}{7}} f_{7}\left(s_{5}\right) \\
\varphi^{\frac{4}{7}} f_{1}\left(s_{5}\right) & \varphi^{\frac{3}{7}} f_{2}\left(s_{4}\right) & \varphi^{\frac{2}{7}} f_{3}\left(s_{3}\right) & \varphi^{\frac{1}{7}} f_{4}\left(s_{2}\right) & f_{5}\left(s_{1}\right) & \varphi^{\frac{6}{7}} f_{6}\left(s_{7}\right) & \varphi^{\frac{5}{7}} f_{7}\left(s_{6}\right) \\
\varphi^{\frac{5}{7}} 1\left(s_{6}\right) & \varphi^{\frac{4}{7}} f_{2}\left(s_{5}\right) & \varphi^{\frac{3}{7}} f_{3}\left(s_{4}\right) & \varphi^{\frac{2}{7}} f_{4}\left(s_{3}\right) & \varphi^{\frac{1}{7}} f_{5}\left(s_{2}\right) & f_{6}\left(s_{1}\right) & \varphi^{\frac{6}{7}} f_{7}\left(s_{7}\right) \\
\varphi^{\frac{1}{7}} f_{1}\left(s_{7}\right) & \varphi^{\frac{2}{7}} f_{2}\left(s_{6}\right) & \varphi^{\frac{3}{7}} f_{3}\left(s_{5}\right) & \varphi^{\frac{4}{7}} f_{4}\left(s_{4}\right) & \varphi^{\frac{5}{7}} f_{5}\left(s_{3}\right) & \varphi^{\frac{6}{7}} f_{6}\left(s_{2}\right) & f_{7}\left(s_{1}\right)
\end{array}\right]
$$

In the case of STBC, a column-wise vectorized form of Eq.(4) is used, which contains the generator matrix of the ST codes as follow:

$$
\begin{gathered}
\mathrm{Y}_{M \times 1}=\operatorname{vec}(\mathrm{Y})=\operatorname{vec}(\mathrm{HX}+\mathrm{N}) \\
\mathrm{Y}_{M \times 1}=\left[\begin{array}{ccc}
\mathrm{H} & 0 & 0 \\
0 & \ddots & 0 \\
0 & 0 & \mathrm{H}
\end{array}\right] \operatorname{vec}(\mathrm{X})+\operatorname{vec}(\mathrm{N}) \\
=\mathrm{H}_{M \times M} \mathrm{G}_{S T} \mathrm{~S}_{M, 1}+\mathrm{N}_{C \times T} \\
=H_{e q} \mathrm{~S}_{M, 1}+\mathrm{N}
\end{gathered}
$$

where $\mathrm{G}_{S T}$ is the generator matrix of the ST code in [27]. $S_{M, 1}=\left[s_{1}, \ldots, s_{M}\right]^{T}$ is the vector of transmitted modulated symbols.

\subsection{ST System Model}

\subsubsection{Decoder}

In this part, we analyze the performance of the optimal ML decoder on the coded MCF transmission system impaired by the CDL. In this case, we observe the effect of ST codes on the BER performance. First, the ML detection criteria of the coded system are given as follows:

$$
\hat{X}_{M L}=\operatorname{argmin}_{X \in \mathcal{C}}\left\|\mathrm{Y}_{M, 1}-H_{e q} \mathrm{X}_{M, 1}\right\|^{2}
$$

where $\mathcal{C}$ is the set of all possible transmitted codewords. Starting from Eq.(10) which describes the pairwise error probability (PEP) of detecting $X_{2}$ given that $X_{1}$ has been transmitted:

$$
\begin{gathered}
\operatorname{Pr}\left\{\mathrm{X}_{1} \rightarrow \mathrm{X}_{2} \mid \mathrm{H}\right\}=Q\left(\frac{\left\|\mathrm{HX} \mathrm{X}_{\Delta}\right\|}{\sqrt{2 N_{0}}}\right) \\
\operatorname{Pr}\left\{\mathrm{X}_{1} \rightarrow \mathrm{X}_{2} \mid \mathrm{H}\right\}=Q\left(\sqrt{\frac{\operatorname{Tr}\left(\mathrm{HX}_{\Delta} \mathrm{X}_{\Delta}^{H} \mathrm{H}^{H}\right)}{2 N_{0}}}\right)
\end{gathered}
$$

After replacing the matrix $\mathrm{H}$ by the corresponding channel model in Eq.(7) and applying the permutation property of the trace, Eq.(44) is given as:

$$
P_{e} \lesssim N_{\min } Q\left(\sqrt{\frac{\ell d_{\min }^{2} \gamma_{0}}{2} \sum_{i} \sum_{j} \lambda_{i}\left|v_{i, j}\right|^{2}}\right)
$$

Having orthogonal closest neighbors $\lambda_{i}$, the double sum can be separated so that the expression can be rewritten as [20]:

$$
P_{e} \lesssim N_{\min } Q\left(\sqrt{\frac{\ell d_{\min }^{2} \gamma_{0}}{2} \sum_{i} \lambda_{i} \sum_{j}\left|v_{i, j}\right|^{2}}\right)
$$

In the case of $C \times C$ TAST coded system, there are $N_{\text {min }}=C^{2} N_{\min }(Q A M)$ codewords at minimum distance $d_{\min }$ which has been proven in [20]. As a simple example, we apply $4-Q A M$ constellation $\left(N_{\min }(Q A M)=2\right)$, each codeword has $N_{\min }(Q A M)=49 \times 2$ closest neighbors at minimum distance $d_{\text {min }}$. $\ell$ can be substituted by its value $\left(\ell=\frac{C}{\sum_{i=1}^{C} \lambda_{i}}\right)$ and the summation of squared elements of the unitary matrix $V$ is equal to 1 . Thus, the upper bound expression in Eq.(46) is given as:

$$
P_{e} \lesssim C^{2} N_{\min } Q\left(\sqrt{\frac{d_{\min \gamma_{0}}^{2}}{2}}\right)
$$

From the previous expression, the CDL can be mitigated by the orthogonality between the nearest neighbors. Moreover, it shows that when the closest neighbors are the dominant term in PEP analysis, TAST codes mitigate the CDL and obtain $C$ Gaussian parallel channels. The last expression shows that MCF system with TAST code is similar to the Gaussian channel.

\subsubsection{ZF Decoder}

In order to obtain an upper bound expression for the $\mathrm{ZF}$ decoder over the MCF transmission system, we can describe the coded MCF channel model as given in [20]:

$$
\widetilde{\mathrm{H}} \triangleq \operatorname{diag}(\mathrm{H}, \ldots, \mathrm{H}) \Phi
$$

where $\Phi$ is the global $M \times M$ unitary matrix [20].

Then, the post-detection SNR can be rewritten as: 


$$
\gamma=\frac{\gamma_{0}}{\left[\mathrm{H}^{H} \mathrm{H}\right]_{i i}^{-1}}
$$

Hence, $\widetilde{\mathrm{H}}^{H} \widetilde{\mathrm{H}}=\Phi^{H} \operatorname{diag}\left(\mathrm{H}^{H} \mathrm{H}, \ldots, \mathrm{H}^{H} \mathrm{H}\right) \Phi$, we obtain:

$$
\begin{array}{r}
\left(\widetilde{\mathrm{H}}^{\mathrm{H}} \widetilde{\mathrm{H}}\right)^{-1}=\frac{1}{\ell} \Phi^{H} \operatorname{diag}\left(\mathrm{V}^{H} \Omega^{-1} \mathrm{~V}, \ldots, \mathrm{V}^{H} \Omega^{-1} \mathrm{~V}\right) \Phi \\
=\frac{1}{\ell} \psi^{\mathrm{H}} \operatorname{diag}\left(\Omega^{-1}, \ldots, \Omega^{-1}\right) \psi
\end{array}
$$

Where $\psi \triangleq \operatorname{diag}(\mathrm{V}, \ldots, \mathrm{V}) \Phi$ is a unitary matrix. Thus, Eq.(49) can be expressed as:

$$
\gamma=\frac{\gamma_{0} \ell}{\sum_{k=1}^{C} \lambda_{k} \sum_{i=1}^{C} \lambda_{i}^{-1}}
$$

The previous equation is described as the ratio between the harmonic and the arithmetic means of the $\lambda_{i}$. Besides, we notice the effect of TAST code by averaging the $V$ from the post-detection SNR equation.

In fact, this ratio in Eq.(51) can be simplify by the orthogonality defect factor (ODF) of $\widetilde{\mathrm{H}}$ which is defined as the product of the norms of its columns over its determinant [20]:

$$
\delta(\widetilde{\mathrm{H}}) \triangleq \frac{\prod_{i=1}^{C}\left\|\widetilde{h_{i}}\right\|}{|\widetilde{\mathrm{H}}|} \geq 1
$$

when the ODF is equal to one, it means that the channel $\widetilde{\mathrm{H}}$ is orthogonal as [20]:

$|\widetilde{\mathrm{H}}|=\ell^{C}|\mathrm{H}|^{C}|\Phi|=\sqrt{\ell^{C^{2}}}|\Lambda|^{\mathrm{C} / 2}|\mathrm{~V}|^{C}=\sqrt{\ell^{\mathrm{C}^{2}}} \prod_{\mathrm{i}=1}^{\mathrm{C}} \lambda_{i}^{C / 2}$

From Eq.(52) and Eq.(53), the authors in [20] proved that the ODF of $\widetilde{\mathrm{H}}$ for $C \times C$ TAST code is given by:

$$
\delta=\frac{\left(\sum_{i=1}^{C} \lambda_{i} / C\right)^{\frac{C^{2}}{2}}}{\prod_{i=1}^{C} \lambda_{i}^{\frac{C}{2}}}
$$

which can be expressed as the $\frac{C^{2}}{2}$ th power of the ratio between the arithmetic and the geometric means of $\lambda_{i}$. The bit error probability with the ZF decoder is given by:

$$
\begin{gathered}
P_{e} \leq E[a Q(\sqrt{\beta \gamma})] \\
\leq E_{\delta}\left[a Q\left(\sqrt{\frac{\ell \beta \gamma_{0}}{\sum_{k=1}^{C} \lambda_{k} \sum_{i=1}^{C} \lambda_{i}^{-1}}}\right)\right]
\end{gathered}
$$

We can substitute the ratio in last expression by the ODF as mentioned in Eq.(52), so Eq.(55) can be rewritten as:

$$
P_{e} \lesssim E_{\delta}\left[a Q\left(\sqrt{\frac{\beta \gamma_{0}}{\delta}}\right)\right]
$$

One can use the maximum of the ODF to give a simple upper bound of the ZF coded system:

$$
P_{e} \lesssim a Q\left(\sqrt{\frac{\beta \gamma_{0}}{\delta_{\max }}}\right)
$$

From the last expression, we note that ZF performance depends on the ODF for the coded system. Hence, the ZF decoder has an optimal performance when the ODF is equal to 1 through the orthogonality of the channel matrix.

\subsection{Simulation Results}

We simulate the $7 \times 7$ TAST code performance for the 7-core Hetero-MCF with applying ML and ZF decoders. In Figure 11, we plot the BER as a function of the SNR for the coding scheme, where we consider 300 fiber sections $(\mathrm{K}=300)$ with $\sigma_{x, y}=7 \% r_{c}$ and $100 \mathrm{~km}$ fiber length. The uncoded system with ZF and ML decoders have SNR penalty equal to $3 \mathrm{~dB}$ and $4 \mathrm{~dB}$, respectively, at $\mathrm{BER}=$ $10^{-4}$. However, we observe that the CDL is completely mitigated using the ML decoder since the performance is the same as the Gaussian channel $(\mathrm{CDL}=$ zero $)$. On the other hand, we notice that the ZF decoder performs worse than the ML decoder, mainly from the orthogonality between the nearest neighbor in the TAST codewords as explained in Eq.(57).

Regarding the TAST code performance efficiency by completely mitigating the CDL, the decoding complexity of the ML decoder is very large compared to the $\mathrm{ZF}$ decoder. In Table 1, we illustrate the complexity of the ML and $\mathrm{ZF}$ decoders for $M \times M$ MIMO systems as shown in works done by E. Awwad et al. and O. Damen et al. [28, 29] and in particular the numerical application for the 7-core MCF. In the ML decoder case, we consider the complexity estimation of the sphere decoder. Noticeably, implementing the ML decoder for full-rate TAST codes in MCF systems with a large number of cores will become very costly and require very high computational effort. Therefore, for the next section, we propose a solution where we can apply the ZF decoder at the receiver side and achieve performance close to the ML decoder and the Gaussian channel.

Table 1. Comparison of the complexities using ML and ZF decoders, $q$ is the number of QAM symbols [28].

\begin{tabular}{lll}
\hline & ZF & ML \\
\hline$M \times M$ MIMO & $\frac{M^{3}+3 M^{2}-M}{M}$ & $\approx q^{M}$ \\
$7 \times 7$ TAST $(M=49)$ & 2547 & $2.6 \times 10^{8}$ \\
\hline
\end{tabular}

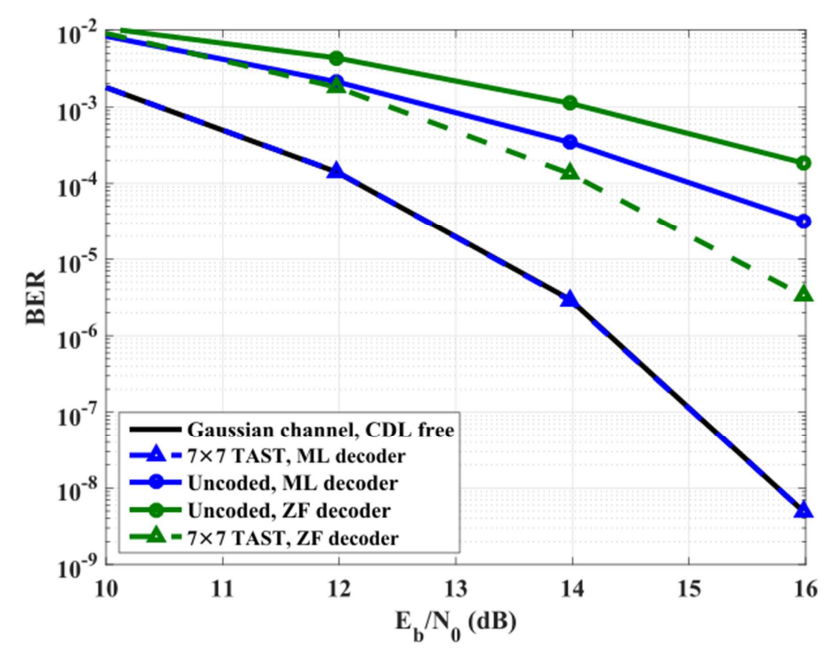

Figure 11. $7 \times 7$ TAST code performance for 7-core Hetero-MCF, misalignment level $\left(\sigma_{x, y}=7 \% r_{c}\right)$. 


\section{Low Complex Detection with ZF}

In this part, we investigate the performance of TAST code combined with core scrambling, aiming to reduce the decoding complexity. The received signal for the coded system is given in Eq. (38) where the singular values follow the same distribution as shown in Figure 8(b). In order to investigate the performance of TAST codes with scrambling, we simulate the $7 \times 7$ TAST code with core scrambling for 7-core Hetero-MCF transmission system as shown in Figure 12. In this scheme, we set the transverse offset to be equal to $7 \% r_{c}$ with $K=300$ and use 6 scramblers in the fiber link, corresponding to $\mathrm{CDL}=1.8 \mathrm{~dB}$. Then, we apply the sub-optimal ZF decoder at the receiver side. In Figure 13, we plot the BER performance and we notice the coding gain provided by the TAST code combined with core scrambling while applying the sub-optimal ZF decoder (dashed line), the system performance with TAST code and core scrambling is enhanced by $1.2 \mathrm{~dB}$ compared to the uncoded system after applying scrambling with ML decoder. In addition, the system performance has only SNR penalty equal to $0.1 \mathrm{~dB}$ compared to the Gaussian channel and the optimal solution $(7 \times 7$ TAST with ML decoder $)$ at $\mathrm{BER}=10^{-4}$.

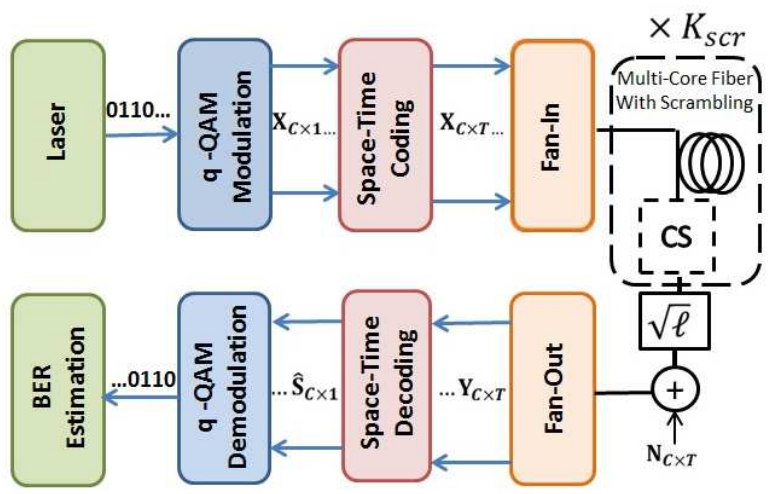

Figure 12. Space-Time coded MCF transmission system with core scrambling.

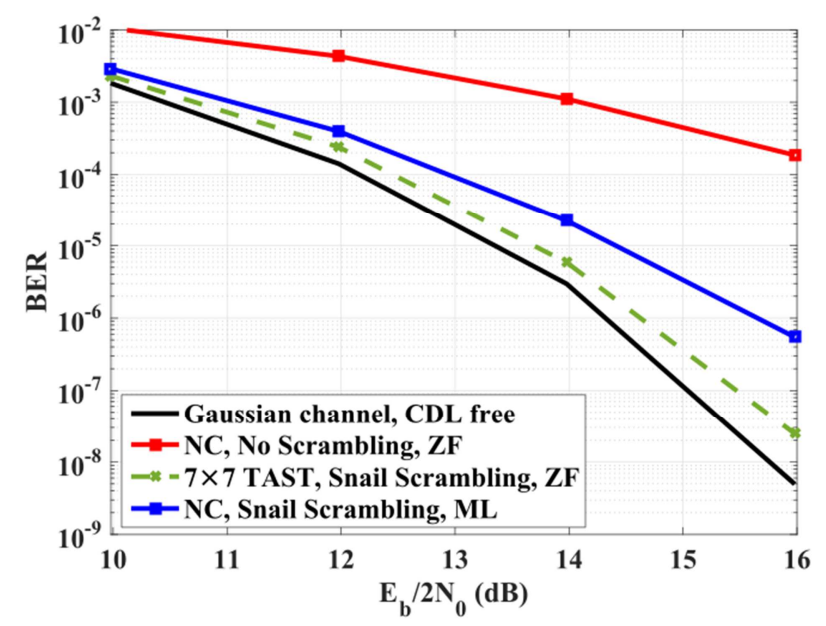

Figure 13. BER performance of 7-core Hetero-MCF with $7 \times 7$ TAST code and core scrambling, with misalignment level $\sigma_{x, y}=7 \% r_{c}$ corresponding to $C D L=1.8 d B$

To explain the previous observation, we need to return to the upper bound on the error probability expression obtained for the ZF decoder in Eq. (57). We proved that the ZF error probability is a function of the ODF where in the case of $\mathrm{ODF}=1$, the channel model is orthogonal and the $\mathrm{ZF}$ performs optimally. Thus, Figure 14 shows the CDF of the ODF $(\delta)$ for the 7-core Hetero-MCF channel model with and without core scrambling. We observe that the system without core scrambling has an orthogonal defect factor that is $70 \%$ of the time equal to 4 . In contrast, the MCF system with core scrambling has an ODF with less than 1.5 during $70 \%$ of the time which makes the ZF near to the optimal performance so close to the ML decoder and Gaussian channel.

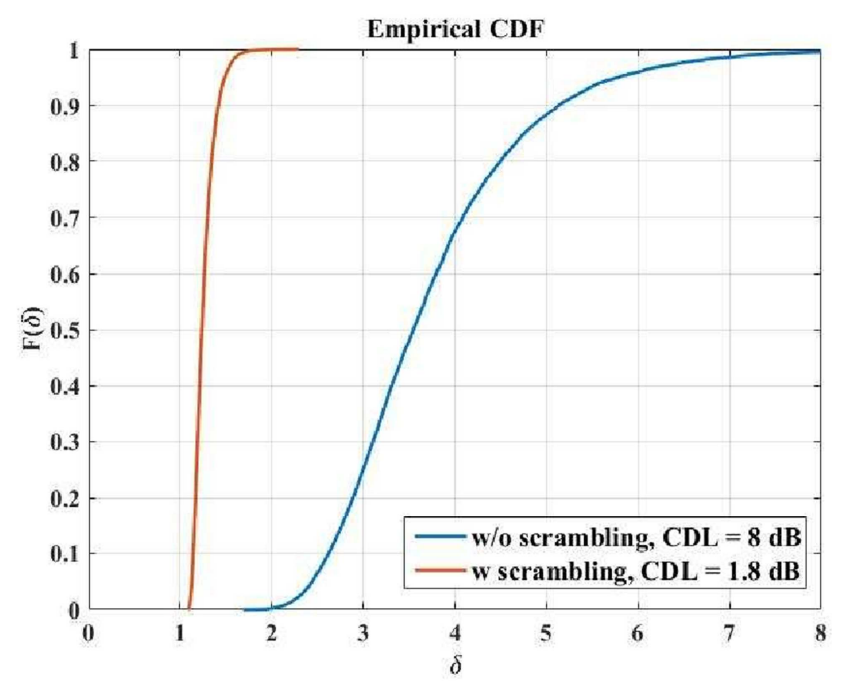

Figure 14. $C D F$ of the $O D F$ for $7 \times 7$ TAST with and without core scrambling

\section{Conclusion}

In this paper, we analyzed the performance of the transmission system of MCFs impaired by CDL under ML and $\mathrm{ZF}$ detection. We established a narrow upper bound on the error probability for both decoders with and without the application of core scrambling. Therefore, we have proved that $\mathrm{ZF}$ and ML have the same upper bound error probability due to the scrambling effect. Since this upper bound is close to the Gaussian channel error performance with SNR reduced by the value of the CDL, it follows that both $\mathrm{ML}$ and $\mathrm{ZF}$ detection transforms the MCF into parallel $\boldsymbol{C}$ Gaussian channels with SNR reduced by the CDL. Then, we proved that TAST codes could completely mitigate the CDL by applying the ML decoder. Further, we showed that we could reduce the decoder complexity of ST coded schemes by applying the sub-optimal ZF decoder with TAST codes and core scrambling. The obtained results show considerable system enhancement with $3.5 \mathrm{~dB}$ SNR gain compare to the uncoded system and performance very close to the Gaussian channel. Moreover, this solution is practical since it allows applying the ZF decoder at the DSP decoding part, which provides a lower coherent receiver cost. In the future, we can analyze the MCF transmission performance after considering the polarization effects such as the PDL. Also, we aim to validate the proposed solution experimentally. 


\section{Appendix}

\section{Appendix A. The PDF of the Maximum and Minimum of Normal Distribution}

The PDF of the maximum and minimum of normal distribution.

We drive the PDF of $U=\max \{X\}$ and $V=\min \{X\}$ where $\mathrm{X}$ is normal distribution with mean $\mu$ and standard deviation $\sigma$

The normal distribution has a CDF:

$$
F(x)=\frac{1}{2}\left[1+\operatorname{erfc}\left(\frac{x-\mu}{\sigma \sqrt{2}}\right)\right]
$$

Assuming $G_{U}(x)$ is the $\mathrm{CDF}$ of $U$ which is equal to $(F(x))^{n}$ where $n$ is the number of sample of independent and identically distributed random variables. Also, $G_{V}(x)$ is the CDF of $V$ which is equal to $1-(1-F(x))^{n}$

The PDF is obtained by taking the derivative of the CDF. In this case, the PDF of $G_{U}(x)=n F(x)^{n-1} f(x)$, which is given as:

$$
f_{U}(x)=\frac{n}{2^{n-2} \sigma \sqrt{2 \pi}}\left[1+\operatorname{erfc}\left(\frac{x-\mu}{\sigma \sqrt{2}}\right)\right]^{n-1} e^{-\left(\frac{x-\mu}{\sqrt{2} \sigma}\right)^{2}}
$$

And the PDF of $G_{V}(x)=n(1-F(x))^{n-1} f(x)$, , knowing that $\operatorname{erfc}(x)=1-\operatorname{erf}(x)$ is:

$$
f_{V}(x)=\frac{n}{\sigma \sqrt{2 \pi}}\left[\operatorname{erfc}\left(\frac{x-\mu}{\sigma \sqrt{2}}\right)\right]^{n-1} e^{-\left(\frac{x-\mu}{\sqrt{2} \sigma}\right)^{2}}
$$

\section{Appendix B. Upper Bound on Error Probability ML Decoder}

Upper Bound on Error Probability ML decoder.

Here we drive the upper bound in Eq.(16). We substitute Eq.(17) in (16) and applying the Chernoff upper bound:

$$
P_{e} \lesssim K \int_{0}^{\infty} e^{-\frac{d_{\min }^{2} \gamma_{0}}{2 \lambda_{\max }} x}\left[\operatorname{erfc}\left(\frac{x-\mu}{\sigma \sqrt{2}}\right)\right]^{C-1} e^{-\left(\frac{x-\mu}{\sqrt{2} \sigma}\right)^{2}} d x
$$

with $K=\frac{C N_{\min }}{\sigma_{z} \sqrt{2 \pi}}$. However, $\operatorname{erfc}(z)$ can be upper bounded by $e^{-z / 2}$. Then, by completing the square after the multiplication of the three exponential terms, Eq.(16) is expressed as:

$$
\begin{gathered}
P_{e} \lesssim K \int_{0}^{\infty} e^{-\frac{d_{\min }^{2} \gamma_{0}}{2 \lambda_{\max }} x} e^{-(C-1)\left(\frac{x-\mu}{\sqrt{2} \sigma}\right)^{2}} e^{-\left(\frac{x-\mu}{\sqrt{2} \sigma}\right)^{2}} d x \\
\lesssim K \int_{0}^{\infty} e^{-C\left(\frac{x-\mu}{\sqrt{2} \sigma}\right)^{2}-\frac{d_{\min }^{2} \gamma_{0}}{2 \lambda_{\max }}} d x \\
\lesssim K e^{\frac{\mu^{2}}{2 \pi \sigma^{2}}}\left[\pi \sqrt{\frac{\sigma_{Z}^{2}}{2 C}} e^{\frac{b}{4 a}} \operatorname{erfc}\left(\frac{1}{2} \sqrt{\frac{b}{a}}\right)\right]
\end{gathered}
$$

where $b=\frac{C d_{\min }^{2} \gamma_{0}}{\lambda_{\operatorname{maz}}}$ and $a=\frac{c}{2 \pi \sigma^{2}}$.

The previous equation can be simplified by upper bounded the erfc(.) to exponential. Also, one can replace $\mu$ by the $\lambda_{\min }$ since it represents the mean value of the minimum eigenvalues. So, the upper bound can be rewritten as:

$$
P_{e} \lesssim \widetilde{K} e^{\frac{\lambda_{\min }^{2}}{2 \pi \sigma^{2}}} e^{-\pi \sigma^{2} \frac{d_{\min }^{2} \gamma_{0}}{2 \lambda_{\max }}}
$$

where $\widetilde{K}=\frac{\pi \sigma}{\sqrt{2 C}}$.

\section{References}

[1] D. Richardson, J. Fini, and L. Nelson, "Space division multiplexing in optical fibers" Nature Photonics, vol. 7, pp. 354-362, 2013.

[2] J. Tu, K. Saitoh, M. Koshiba, K. Takenaga, and S. Matsuo, "Design and analysis of large-effective-area heterogeneous trench-assisted multi-core fiber," Optics express, vol. 20, no. 14, pp. 15157-15170, 2012.

[3] Hayashi, T. Taru, O. Shimakawa, T. Sasaki, and E. Sasaoka, "Design and fabrication of ultra-low crosstalk and low-loss multi-core fiber," Optics express, vol. 19, no. 17, pp. 1657616592, 2011.

[4] Mizuno, K. Shibahara, H. Ono, Y. Abe, Y. Miyamoto, F. Ye, T. Morioka, Y. Sasaki, Y. Amma, and K. Takenaga, "32-core dense SDM unidirectional transmission of PDM-16QAM signals over $1600 \mathrm{~km}$ using crosstalk-managed single-mode heterogeneous multicore transmission line," in IEEE Optical Fiber Communications Conference and Exhibition, pp. 1-3, 2016.

[5] T. M. F. Alves and A. V. T. Cartaxo, "Experimental and analytical characterization of time variation of ICXT in MCFs with multiple interfering cores," in Optical Fiber Communications Conference and Exposition (OFC), pp. 1-3, 2018.

[6] M. Koshiba, K. Saitoh, K. Takenaga, and S. Matsuo, "Multi-core fiber design and analysis: coupled-mode theory and coupled-power theory," Optics express, vol. 19, no. 26, pp. B102-B111, 2011.

[7] K. Watanabe and T. Saito, "Compact fan-out for 19-coremulticore fiber with high manufacture ability and good optical properties," in IEEE Opto-Electronics and Communications Conference, 2015, pp. 1-3, 2015.

[8] A. Abouseif, G. Rekaya Ben-Othman, and Y. Jaouën, "Channel model and optimal core scrambling for multi-core fiber transmission system," Optics Communications, vol. 454, p. 124396, 2020.

[9] K.-P. Ho and J. M. Kahn, "Mode-dependent loss and gain: statistics and effect on mode-division multiplexing," Opt. Express, vol. 19, pp. 16612-16635, Aug 2011.

[10] S. Warm and K. Petermann, "Splice loss requirements in multi-mode fiber mode-division-multiplex transmission links,"Optics express, vol. 21, no. 1, pp. 519-532, 2013.

[11] S. Luis, B. J. Puttnam, G. Rademacher, Y. Awaji, and N. Wada, "On the use of high-order MIMO for long-distance homogeneous single-mode multicore fiber transmission," in European Conference on Optical Communication (ECOC), pp. 1-3, IEEE, 2017.

[12] Filipenko and O. Sychova, "Research of misalignments and cross-sectional structure influence on optical loss in photonic crystal fibers connections," in IEEE International Conference on Advanced Optoelectronics and Lasers, pp. 85-87, 2013. 
[13] D. Marcuse, "Loss analysis of single-mode fiber splices," Bell System Technical Journal, vol. 56, no. 5, pp. 703-718, 1977.

[14] J. Cui, S. Zhu, K. Feng, D. Hong, J. Li, and J. Tan, "Fan-out device for multicore fiber coupling application based on capillary bridge self-assembly fabrication method,"Optical Fiber Technology, vol. 26, pp. 234-242, 2015.

[15] P. J. Winzer and G. J. Foschini, "MIMO capacities and outage probabilities in spatially multiplexed optical transport systems," Optics Express, vol. 19, no. 17, pp. 16680-16696, 2011.

[16] Ryf, S. Randel, A. H. Gnauck, C. Bolle, A. Sierra, S. Mumtaz, M. Esmaeelpour, E. C. Burrows, R.-J. Essiambre, P. J. Winzer, et al., "Mode-division multiplexing over $96 \mathrm{~km}$ of few-mode fiber using coherent $6 \times 6$ MIMO processing," Journal of Light wave technology, vol. 30, no. 4, pp. 521-531, 2012.

[17] B. Inan, B. Spinnler, F. Ferreira, D. van den Borne, A. Lobato, S. Adhikari, V. A. Sleiffer, M. Kuschnerov, N. Hanik, and S. L. Jansen, "DSP complexity of mode-division multiplexed receivers," Optics express, vol. 20, no. 10, pp. 10859-10869, 2012.

[18] R. Epworth, "Phenomenon of modal noise in fiber systems," in Optical Fiber Communication Conference, p. ThD1, Optical Society of America, 1979.

[19] M. K. Simon and M.-S. Alouini, Digital communication over fading channels, vol. 95. John Wiley \& Sons, 2005.

[20] Damen and G. Rekaya-Ben Othman, "On the performance of spatial modulations over multimode optical fiber transmission channels," IEEE Transactions on Communications, vol. 67, pp. 3470-3481, May 2019.

[21] L. Bachioua, "Extended lognormal distribution: Properties and applications," The Scientific World Journal, 2020.
[22] J. Proakis, Digital Communications, 4th edition. McGraw-Hillseries in Electrical and Computer Engineering, 2000.

[23] R. Xu and F. Lau, "Performance analysis for MIMO systems using zero forcing detector over fading channels," IEEE Proceedings-Communications, vol. 153, no. 1, pp. 74-80, 2006.

[24] C. R. Schwarz, "Statistics of range of a set of normally distributed numbers," Journal of surveying engineering, vol. 132, no. 4, pp. 155-159, 2006.

[25] E.-M. Amhoud, G. Rekaya Ben-Othman, and Y. Jaouën, "Concatenation of space-time coding and FEC for few-mode fiber systems," IEEE Photonics Technology Letters, vol. 29, pp. 603-606, 2017.

[26] V. Tarokh, N. Seshadri, and A. R. Calderbank, "Space-time codes for high data rate wireless communication: Performance criterion and code construction," IEEE transactions on information theory, vol. 44, no. 2, pp. 744-765, 1998.

[27] H. El Gamal and M. O. Damen, "Universal space-time coding," IEEE Transactions on Information Theory, vol. 49, no. 5, pp. 1097-1119, 2003.

[28] E. Awwad, G. Rekaya Ben-Othman, and Y. Jaouën, "Space-time coding schemes for MDL-impaired mode-multiplexed fiber transmission systems," Journal of Lightwave Technology, vol. 33, no. 24, pp. 5084-5094, 2015.

[29] O. Damen, A. Chkeif, and J.-C. Belfiore, "Lattice code decoder for space-time codes," IEEE Communications letters, vol. 4, no. 5, pp. 161-163, 2000. 